\title{
Aplicação de ferramenta computacional para monitoramento do comportamento de um processo químico
}

\author{
Adalício Uzêda Antunes Júnior* (Mestrando em Engenharia Química na Universidade Federal de \\ Campina Grande - UFCG);
}

José Mariano da Silva Neto (Graduando em Engenharia Química na Universidade Federal de Campina

$$
\text { Grande - UFCG); }
$$

Maria Isabel do Bú Araújo (Graduanda em Engenharia Química na Universidade Federal de Campina

$$
\text { Grande - UFCG); }
$$

Maria Rosiane de Almeida Andrade (Graduanda em Engenharia Química na Universidade Federal de Campina Grande - UFCG).

*E-mail: adaliciouzeda@hotmail.com

\section{Resumo:}

O uso de ferramentas computacionais no âmbito acadêmico proporciona uma expansão do conhecimento dos discentes através da simulação de diferentes cenários de determinados processos e a aplicação de conceitos fundamentais. A evolução constante da tecnologia e desenvolvimento de novos produtos cada vez mais completos e eficientes tem possibilitado uma maior praticidade na área de modelagem e simulação de processos desde processos comuns até complexos utilizados em sistemas industriais.

O intuito desta pesquisa é de analisar a influência do uso de ferramentas computacionais para resolução de problemas e verificar a eficiência desta prática para entendimento e interpretação do processo adotado. Neste trabalho, utilizou-se o software MATLAB ${ }^{\circledR}$, amplamente utilizado no meio acadêmico e de setor de pesquisa, na avaliação do comportamento dinâmico de variáveis em um processo fermentativo em biorreator.

Através desta abordagem, pôde-se analisar a influência de fatores no processo fermentativo em um biorreator com acompanhamento contínuo de biomassa, produto e substrato ao longo do tempo. Foram considerados diversos parâmetros adotados na modelagem matemática para ser variados e foi verificado o impacto das modificações destes parâmetros na resposta do processo fermentativo. Pôde-se, além disso, avaliar a rapidez do equilíbrio alcançado e comportamento dinâmico das concentrações das variáveis de interesse.

A ferramenta mostra-se eficiente para verificação dos parâmetros considerados e facilita o entendimento do processo escolhido, bem como, auxilia na interpretação de resultados de problemas através da análise crítica para resolução de desafios. É uma facilitadora da aplicação de conceitos básicos aprendidos pelo usuário e poderosa alternativa pela sua praticidade de uso e rapidez na obtenção de resultados. 


\section{Aplicação de ferramentas computacionais}

O desenvolvimento das ferramentas computacionais possibilita a expansão de uma fronteira até então pouco explorada, a da incorporação da modelagem e simulação como método de ensino (BELHOT et al., 2001). O principal intuito desta aplicação deve-se ao fato da busca da expansão do entendimento sobre conceitos aplicados e capacidade de análise através da interpretação do comportamento de determinado processo químico.

O uso da modelagem matemática e da simulação de processos são de fundamental importância nos campos de pesquisa e desenvolvimento, de projeto e de operação. A validação da modelagem matemática junto a dados experimentais, estudo de condições operacionais para otimização e controle, análise da operabilidade dos processos, possibilidade de verificar diferentes cenários, avaliação de situações de start up e shut down, treinamento de operadores e acompanhamento geral das variáveis envolvidas são algumas das principais vantagens para a aplicação das ferramentas computacionais na análise de um processo.

A modelagem matemática, em conjunto a simulação de processos são poderosas ferramentas para o estudo e entendimento do comportamento de processos complexos comuns da Engenharia Química e áreas afins. Estas ferramentas já encontram grande aplicação no meio industrial e no campo da pesquisa, porém ainda não são muito utilizadas no meio acadêmico como um método sistematizado para o ensino (PELISSON e VIEIRA, 2015).

Uma etapa essencial no desenvolvimento de qualquer modelo é a formulação das equações de balanço de massa e energia, podendo-se incluir relações adicionais que representem: as taxas de reação química, as taxas de transferência de calor e massa e alterações de propriedades do sistema. A combinação destas relações constitui a base para a descrição quantitativa do processo e compreende o modelo matemático básico. O modelo resultante pode variar de um simples caso de equações até modelos de grande complexidade. Uma das habilidades da modelagem é, portanto, obter o modelo mais simples possível que seja capaz de uma representação realista do processo (INGHAM et al., 2007).

De acordo com Luyben (1996), a modelagem matemática de um sistema proporciona um auxílio no diagnóstico de problemas de controle e processo, gera a capacitação dos envolvidos na atividade, permite a análise da otimização para melhorias na qualidade, produtividade, sustentáveis e lucrativas, além do fato de ser uma metodologia barata e mais rápida quando comparada a testes experimentais.

O desenvolvimento da modelagem matemática por parte do educando é de fundamental importância. Através de ferramentas deste tipo de programa, os alunos podem descrever relações entre conceitos, aplicar os modelos construídos e comparar os resultados obtidos com o conhecimento que é aceito pela comunidade científica ou com experimentos laboratoriais. Através de condições proporcionadas por novas tecnologias, como softwares de simulação, alunos podem isolar variáveis, estudar seus padrões e comportamentos e, a partir disto, encontrar as relações, os princípios e as leis que regem o fenômeno estudado (RIBEIRO E GRECA, 2003).

O estudo da modelagem matemática permite prever o comportamento dinâmico e estacionário do processo, inclusive em condições não testadas empiricamente, possibilitando a determinação de condições operacionais economicamente ótimas do sistema, avaliando o projeto e o ajuste de algoritmos de controle, no qual o modelo matemático formulado passa a ser parte integrante do mesmo (INGHAM et al., 2007).

Os simuladores auxiliam os engenheiros a projetar o processo e a planta industrial. Estes programas possuem a capacidade de construir diagramas de processo e instrumentação, os quais podem ser dinâmicos, realizando balanços de massa e energia, além de serem configurados para receber sinais da planta e mostrar o valor das variáveis de processo em tempo real, assim como, enviar comandos de atuação para modificar estas variáveis (LEITE et al. 2014).

Dentre os diversos softwares que são aplicados como ferramentas computacionais em modelagem e simulação de processos, o $M A T L A B^{\circledR}$ tem destaque pela sua praticidade e capacidade de uso em diversas áreas. Os estudos desenvolvidos por Mariani e Martim (2003), Dias et al. (2013) e Breitkreitz et al. (2013) enfatizam a importância do contato do estudante de graduação com o software MATLAB ${ }^{\circledR}$ para a resolução de problemas do cotidiano na área da Química e Engenharia Química. Verificaram ainda a necessidade dos discentes sobre o uso de 
ferramentas computacionais para melhor assimilar os conteúdos e promover análise crítica de problemas.

As modelizações computacionais em Química são fundamentais, por se tratar de uma Ciência que se constrói a partir da exploração do invisível e intocável, constituindo um estudo inerentemente simbólico e representativo (KOZMA E RUSSELL, 1997).

Diante disso, o intuito deste trabalho é aplicar o uso de ferramentas computacionais para que seja ampliado o conhecimento e possa facilitar o entendimento de um processo fermentativo em reator batelada.

\section{Processos fermentativos em biorreator}

Um dos objetivos da modelagem matemática e simulação, como ferramenta do desenvolvimento tecnológico de processos fermentativos, é prever o comportamento dinâmico e estacionário do processo, inclusive em condições não testadas empiricamente, possibilitando a determinação das condições operacionais economicamente ótimas do sistema, auxiliando no projeto e ajuste de algoritmos de controle, no qual o modelo matemático formulado passa a ser parte integrante do mesmo (BORZANI et al., 2001).

Um biorreator é um recipiente que tem como objetivos: conter o leito de substrato, impedir a liberação do microrganismo para as vizinhanças do sistema, impedir a entrada de contaminantes no sistema e manter as condições ótimas para crescimento do microrganismo e formação de produto em seu interior de maneira uniforme, ao longo do processo (MENEZES, 2006).

Os processos fermentativos podem ser classificados como: batelada, batelada alimentada e contínuo. No processo em batelada todos os componentes são inseridos no biorreator no início do processo e não há retirada antes do término do ciclo completo de fermentação. Para o caso em batelada alimentada, há a adição do substrato durante o ciclo para controle da velocidade da reação e reduzir o efeito inibitório resultante do excesso de substrato inicial da etapa. No processo contínuo há um fluxo de alimentação do biorreator, e, simultaneamente, a remoção dos produtos de modo a manter o volume constante no biorreator.

Adotou-se no presente trabalho um biorreator do tipo batelada para a análise do comportamento da concentração de enzima, substrato e produto ao longo da reação fermentativa.

As vantagens da aplicação do uso em batelada, conforme sugerido por Schmidell e Facciotti (2001), deve-se ao fator de ser o processo mais seguro quando se tem problema de manutenção e condições de assepsia, uma vez que, após o término de cada batelada pode-se fazer a esterilização do biorreator e controles necessários no inóculo. Além disso, a operação em batelada apresenta grande flexibilidade de operação.

Como desvantagens, a fermentação descontínua (batelada) pode levar a baixos rendimentos e/ou produtividades, quando o substrato é adicionado de uma só vez no início da fermentação, pois este pode exercer efeitos de inibição, repressão ou desviar o metabolismo celular a produtos que não interessam. Além disso, este tipo de operação apresenta tempos mortos, tais como tempo de carga e descarga e período correspondente a lavagem e esterilização do biorreator (BORZANI et al., 2001).

\section{Metodologia}

A modelagem matemática e parâmetros do processo fermentativo foram baseados em Seeborg et al. (2003). Trata-se de um problema para verificação do monitoramento do comportamento dinâmico da biomassa, substrato e produto ao longo do ciclo de fermentação.

As biorreações, de maneira geral, podem ser caracterizadas pelas reações em que há a alimentação de substrato com biomassa e geram produtos juntamente com o aumento da biomassa no processo fermentativo. Para simplificar o processo, considerou-se que os substratos possuem apenas um nutriente limitante e gera-se apenas um produto.

Para o presente processo, foram utilizadas ainda as seguintes simplificações: biorreator de mistura perfeita, desprezaram-se os efeitos térmicos, a densidade do líquido é constante e o 
sistema considerado como homogêneo, o crescimento exponencial como taxa de crescimento da biomassa e proporcional à concentração de células. A taxa de crescimento específica $(\mu)$ adotada foi baseada na Equação de Monod conforme representada na Equação 1 a seguir.

$$
\mu=\mu_{\text {máx }} \frac{S}{K_{S}+S}
$$

Em que: $\mu_{\text {máx }}$ refere-se ao máximo valor da taxa de crescimento, $S$ representa a quantidade de substrato e o $\mathrm{K}_{\mathrm{s}}$ é denominado de constante de Monod.

A equação de Monod possui a mesma forma da equação de Michaelis-Menten, que representa a expressão padrão para taxa de reação de enzimas (BAILEY e OLLIS, 1986).

De acordo com Fogler (1999), a taxa de crescimento da biomassa por unidade de volume $\left(\mathrm{r}_{\mathrm{g}}\right)$ e a taxa de formação de produto por unidade de volume $\left(\mathrm{r}_{\mathrm{p}}\right)$ são representadas de acordo com a Equação 2 e a Equação 3.

$$
\begin{gathered}
r_{g}=\mu X \\
r_{p}=Y_{P / X} r_{g}
\end{gathered}
$$

O coeficiente de rendimento que relaciona a biomassa formada e substrato consumido é identificado por $\mathrm{Y}_{\mathrm{X} / \mathrm{S}}$. Enquanto que $\mathrm{Y}_{\mathrm{P} / \mathrm{S}}$ representa a interação entre o produto formado e substrato consumido e o $\mathrm{Y}_{\mathrm{P} / \mathrm{X}}$ representa a interação entre o produto formado e biomassa.

Os modelos dinâmicos para representar os balanços de massa em um biorreator batelada para as células da biomassa (X), produto (P) e substrato (S), baseados em Seeborg et al. (2003), são dados por:

$$
\begin{gathered}
\frac{d X}{d t}=r_{g} \\
\frac{d P}{d t}=r_{p} \\
\frac{d S}{d t}=\left(-\frac{r_{g}}{Y_{X / S}}\right)+\left(-\frac{r_{p}}{Y_{P / S}}\right)
\end{gathered}
$$

\section{Resultados e Discussão}

As condições iniciais para concentrações da biomassa, do substrato e do produto adotadas para o presente trabalho seguem apresentadas na Tabela 1.

Tabela 1 - Condições iniciais para os componentes envolvidos no biorreator.

\begin{tabular}{cc}
\hline Componente & Condição inicial \\
\hline Biomassa & $\mathrm{X}(0)=0,05 \mathrm{~g} \cdot \mathrm{L}^{-1}$ \\
Substrato & $\mathrm{S}(0)=10,00 \mathrm{~g} . \mathrm{L}^{-1}$ \\
Produto & $\mathrm{P}(0)=0,00 \mathrm{~g} \cdot \mathrm{L}^{-1}$ \\
\hline
\end{tabular}

Fonte: Seeborg et al. (2003)

Além disso, os demais parâmetros adotados para o processo fermentativo seguem apresentados na Tabela 2.

Tabela 2 - Valores dos parâmetros adotados para o processo fermentativo. 


\begin{tabular}{cc}
\hline Parâmetro & Valor \\
\hline Taxa máxima de crescimento da biomassa & $\mu_{\text {máx }}=0,2 \mathrm{~h}^{-1}$ \\
Constante de Monod & $K_{S}=1,0 \mathrm{~g} \cdot \mathrm{h}^{-1}$ \\
Coeficiente de rendimento da biomassa em relação ao & $Y_{X / S}=0,5 \mathrm{~g} \cdot \mathrm{g}^{-1}$ \\
substrato & $Y_{P / S}=0,1 \mathrm{~g} \cdot \mathrm{g}^{-1}$ \\
Coeficiente de rendimento do produto em relação ao substrato & $Y_{P / X}=0,2 \mathrm{~g} \cdot \mathrm{g}^{-1}$ \\
\hline
\end{tabular}

Fonte: Seeborg et al. (2003)

Após a modelagem matemática, condições iniciais e parâmetros do processo, pode-se obter a análise do comportamento dinâmico da biomassa, substrato e produto. Encontra-se na Gráfico 1 a verificação do desempenho do processo fermentativo.

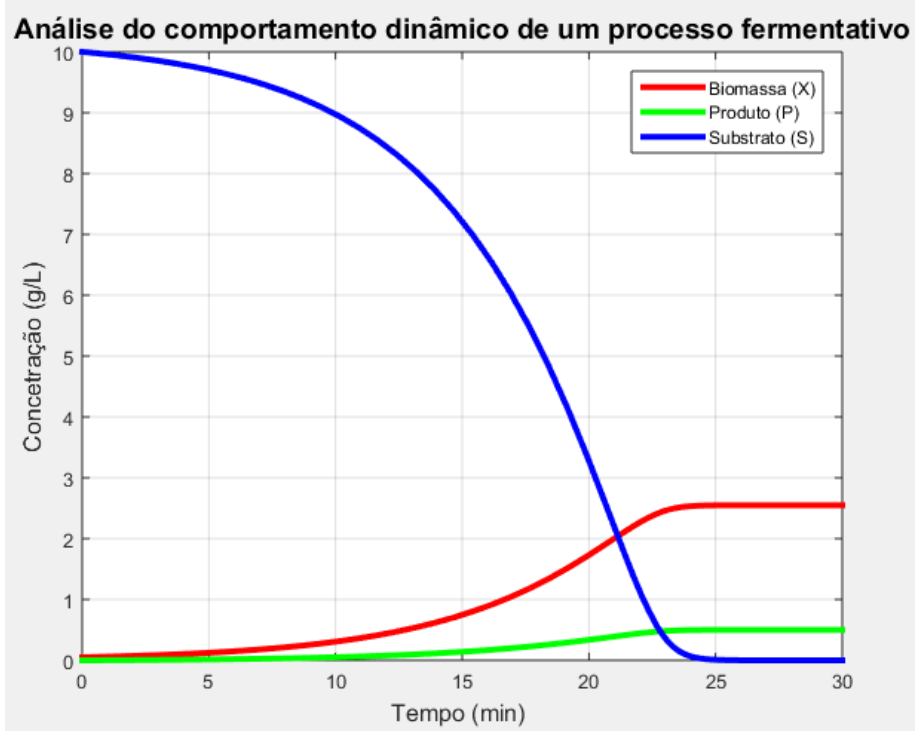

Gráfico 1 - Comportamento do processo fermentativo para biomassa, substrato e produto.

A partir do resultado observado no Gráfico 1, verifica-se que há uma redução da quantidade de substrato desde o valor inicial até o valor zero em um tempo de 25 minutos. Além disso, observa-se que ao longo do tempo há uma produção de biomassa em relação ao valor inicial e que pode ser obtida uma pequena quantidade de produto. Observa-se que por volta de 25 minutos os componentes atingem um valor constante e permanecem assim nos próximos intervalos de tempo.

Com o intuito de entender a influência da quantidade de biomassa no processo, fez-se uma alteração da condição inicial de 0,05 g. $L^{-1}$ para 0,5 g.L $L^{-1}$ e o comportamento segue representado no Gráfico 2.

Verifica-se que houve um aumento no valor da concentração da biomassa no processo fermentativo ao aumentar em 10 vezes a concentração inicial. $O$ valor no equilíbrio para a concentração de biomassa passa de 2,55 g.L $\mathrm{L}^{-1}$ para 3,00 g.L $\mathrm{L}^{-1}$. Em relação ao substrato, verificouse que há um decaimento mais nítido quando comparado ao caso anterior e o estado do equilíbrio é obtido em 12 minutos. Embora o estado de equilíbrio para o produto tenha sido alçado mais rapidamente do que o caso com as considerações iniciais, o valor final para a concentração do produto permaneceu o mesmo. 


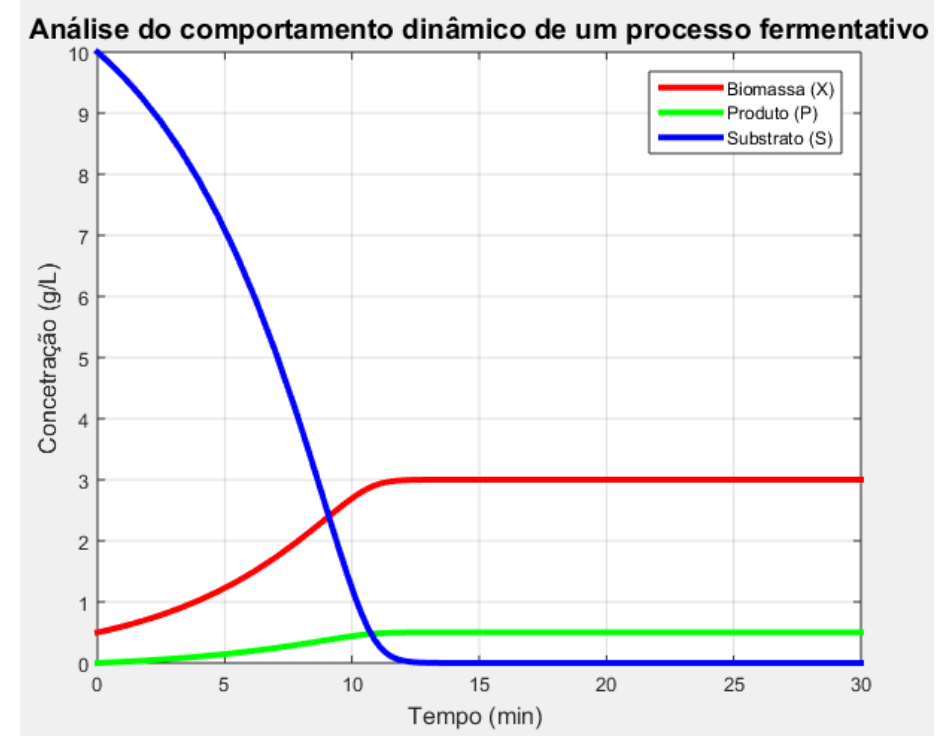

Gráfico 2 - Comportamento do processo fermentativo com modificação na quantidade inicial de biomassa $\left(\mathrm{X}(0)=0,5 \mathrm{~g} \cdot \mathrm{L}^{-1}\right)$.

Observou-se também, conforme constatado no Gráfico 3, a influência do máximo valor da taxa de crescimento $\left(\mu_{\text {máx }}\right)$ no processo fermentativo, passando o valor de $0,2 \mathrm{~h}^{-1}$ para $1 \mathrm{~h}^{-1}$.

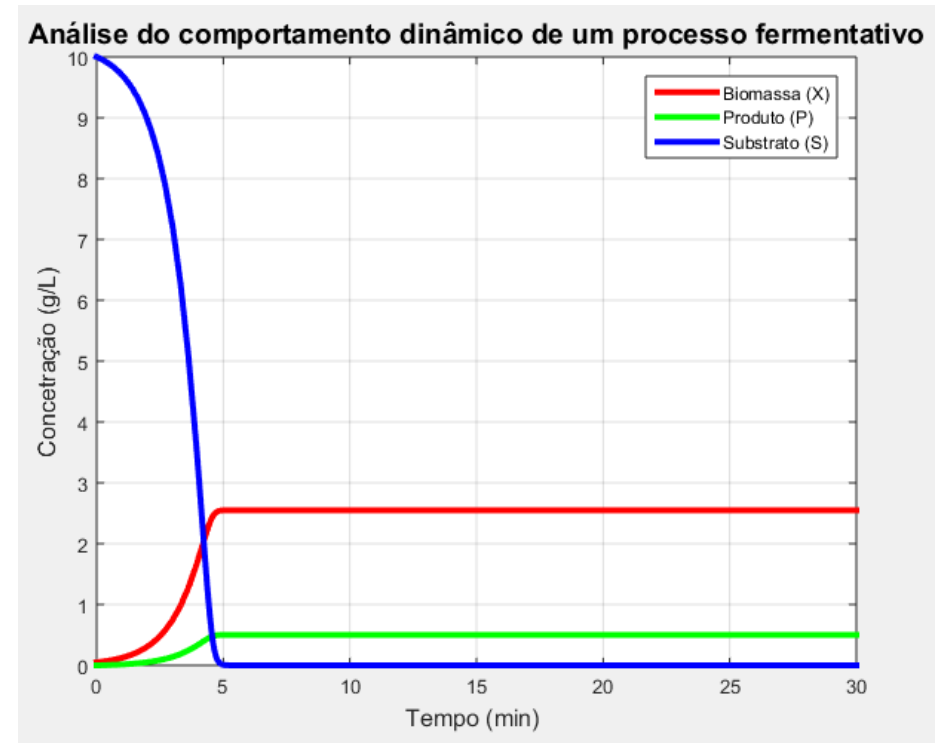

Gráfico 3 - Comportamento do processo fermentativo com modificação do máximo valor da taxa de crescimento $\left(\mu_{\text {máx }}=1\right)$.

Verificou-se que o aumento da taxa máxima de crescimento não alterou os valores finais alcançados no equilíbrio para a biomassa, produto e substrato. No entanto, contata-se que o equilíbrio foi atingido mais rapidamente do que o caso inicial com um tempo de ciclo fermentativo de 5 minutos. Tal fato constata que o aumento deste parâmetro tem influência direta apenas na rapidez da dinâmica do processo. Além disso, a redução deste parâmetro faz com que o estado de equilíbrio seja atingido em um tempo superior.

No Gráfico 4, Gráfico 5 e Gráfico 6, seguem representados os comportamentos para verificar a influência dos parâmetros de rendimento de produto em relação ao substrato e do produto em relação à biomassa. 


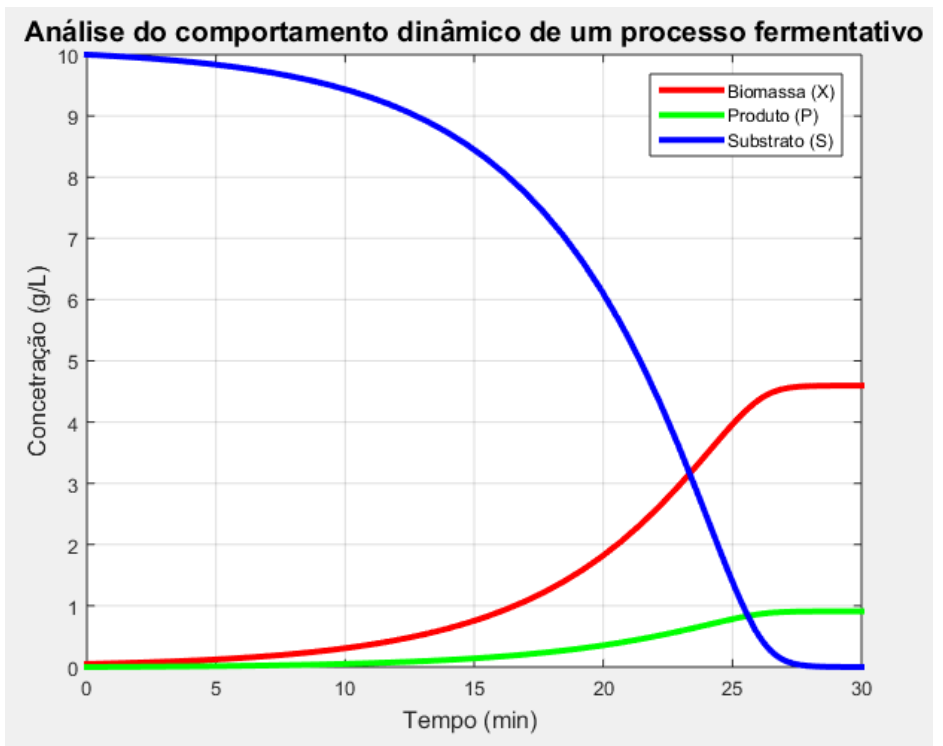

Gráfico 4 - Comportamento do processo fermentativo com modificação do rendimento de produto em relação ao substrato $\left(\mathrm{Y}_{\mathrm{P} / \mathrm{S}}=1\right)$.

Com base nos resultados do Gráfico 4, verifica-se que ao considerar $\mathrm{Y}_{\mathrm{P} / \mathrm{S}}=1$, em relação ao caso inicial, houve aumento da quantidade de biomassa e produto gerados, conseguindo concentrações aproximadas de 4,6 g.L $\mathrm{L}^{-1}$ e 0,91 g.L $\mathrm{L}^{-1}$, respectivamente. Adicionalmente, verificouse que o equilíbrio demorou mais a ser alcançado.

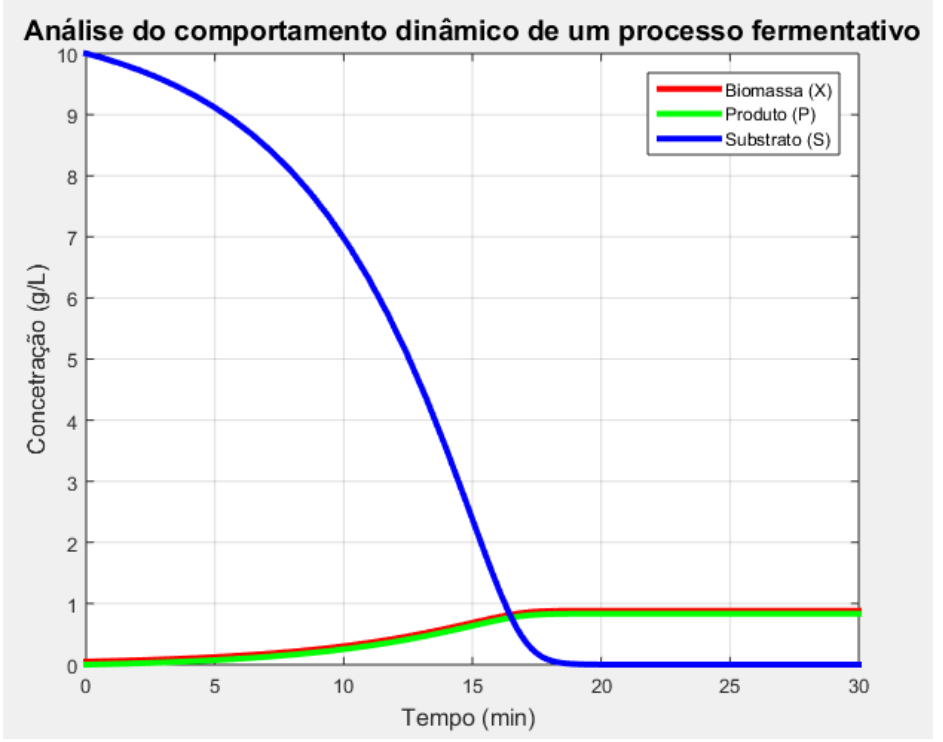

Gráfico 5 - Comportamento do processo fermentativo com modificação do rendimento de produto em relação à biomassa $\left(\mathrm{Y}_{\mathrm{P} / \mathrm{X}}=1\right)$.

A partir dos resultados observados no Gráfico 5, ao considerar $\mathrm{Y}_{\mathrm{P} / \mathrm{X}}=1$, observou-se que promoveu concentrações finais com mesmo valor para o produto e substrato de 0,83 g. $\mathrm{L}^{-1}$. Além disso, o equilíbrio do processo fermentativo foi alcançado mais rapidamente do que no caso inicial considerado.

Ao considerar $\mathrm{Y}_{\mathrm{P} / \mathrm{S}}=1$ e $\mathrm{Y}_{\mathrm{P} / \mathrm{X}}=1$, simultaneamente, conforme apresentado no Gráfico 6, verificou-se que houve aumento das concentrações de produto e biomassa atingindo um valor final próximos de 3,38 g.L $L^{-1}$. Além disso, contatou-se equilíbrio alcançado num tempo bem semelhante ao caso inicial. 


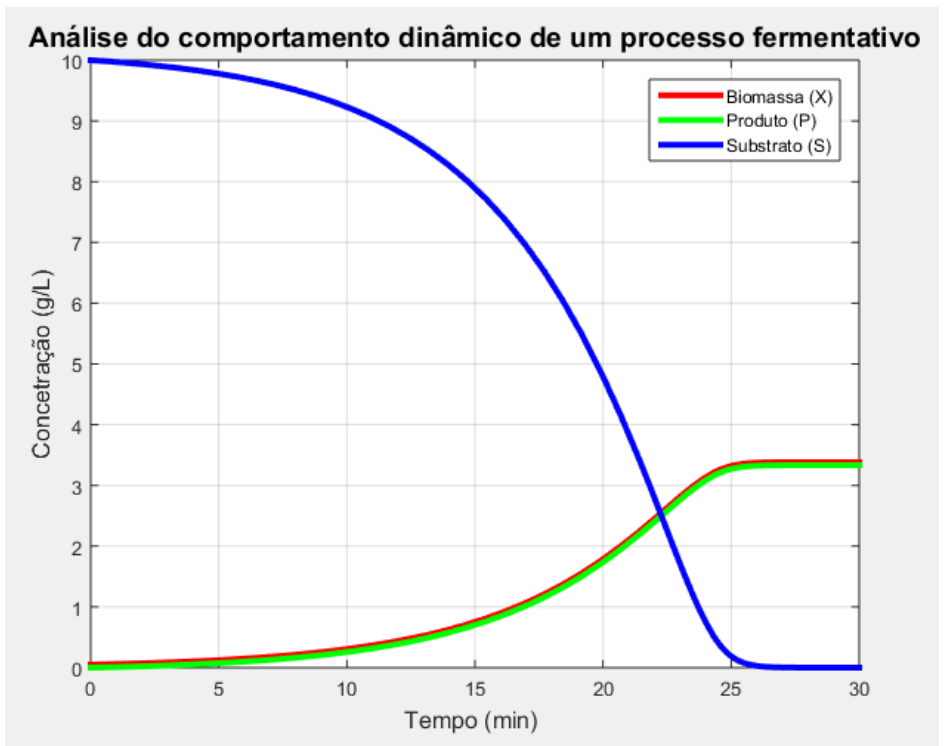

Gráfico 6 - Comportamento do processo fermentativo com modificação dos rendimentos de produto em relação ao substrato $\left(\mathrm{Y}_{\mathrm{P} / \mathrm{S}}=1\right)$ e de produto em relação à biomassa $\left(\mathrm{Y}_{\mathrm{P} / \mathrm{X}}=1\right)$.

O principal intuito da aplicação deste problema foi analisar a capacidade da ferramenta computacional em relação a um determinado processo. A modelagem inserida ao software MATLAB $^{\circledR}$ possibilitou o acompanhamento do comportamento dinâmico das variáveis de concentração de produto, biomassa e substrato. Além disso, de maneira rápida, pode-se obter comportamento previsível para o processo fermentativo ao alterar parâmetros adotados. Os valores das modificações serviram apenas como estudo comparativo em relação às considerações iniciais e não foi realizado um estudo prévio dos exatos valores a ser adotados para cada parâmetro modificado, uma vez que, o intuito principal deste trabalho é avaliar a influência do comportamento dinâmico do processo ao ser submetido algumas alterações.

Em estudo desenvolvido por Janeczko et al. (2012), avaliou-se a influência do tipo de modelagem matemática adotada para análise do comportamento dinâmico da produção de caratenoides em um biorreator em batelada. Utilizou-se no estudo o software MATLAB ${ }^{\circledR}$ para obtenção dos resultados e ressalta a necessidade do estudo da modelagem matemática de processos com biorreatores.

A aplicação das ferramentas computacionais foi também observada em estudos desenvolvidos por Diehl (2009) em que há a análise de um biorreator contínuo de biossíntese de etanol sob a abordagem de controle e otimização.

Em estudos realizados por Condorena (2012), fez-se a análise da produção de etanol de primeira e segunda geração com a utilização de softwares, como o MATLAB ${ }^{\circledR}$, para aplicar estratégias de redes neurais com controle MPC, desenvolver modelagem do processo e realizar a otimização do sistema reacional.

Através da ferramenta computacional aplicada ao processo fermentativo avaliaram-se os valores finais de concentração a ser alcançados no equilíbrio para produto, substrato e biomassa e a rapidez com que o equilíbrio pode ser alcançado em cada um dos casos adotados. $\mathrm{O}$ uso da modelagem matemática e resolução através do software MATLAB ${ }^{\circledR}$ permite um entendimento mais facilitado sobre o comportamento do processo fermentativo e a influência de alguns parâmetros neste processo. Adicionalmente, o usuário pode ser capaz de alterar todos os parâmetros do processo fermentativo e ser capaz de avaliar qual a melhor configuração a ser adotada.

\section{Conclusão}

Diante do exposto, constata-se a importância do uso de ferramentas computacionais, tais como o MATLAB $^{\circledR}$, para auxiliar no entendimento de processos químicos. Através do software utilizado, 
verificou-se a influência de diversas variáveis do processo fermentativo no comportamento dinâmico da biomassa, substrato e produto. A eficiência da ferramenta computacional mostra-se como promissora estratégia para ampliação das metodologias de ensino, facilitadora para aplicação de conceitos práticos adquiridos e difusora da promoção de pensamento crítico e analítico na resolução de problemas na área de processos químicos.

\section{Computational tool application for behavior monitoring of a chemical process}

\section{Abstract:}

The use of computational tools in the academic field provides the expansion of knowledge of students by simulating different scenarios of several processes and the application of fundamental concepts. The constant evolution of technology and development of new products increasingly complete and efficient has enabled a more practical in the sector of modeling and simulation of processes from common to complex processes used in industrial systems.

The purpose of this research is to analyze the influence of the use of computational tools to solve problems and check the effectiveness of this practice for understanding and interpreting the process adopted. In this work, we used the MATLAB ${ }^{\circledR}$ software, widely used in academic and research sector, the assessment of the dynamic behavior of variables in a fermentation bioreactor.

Through this approach, it was possible to analyze the influence of factors in the fermentation process in a bioreactor with continuous monitoring of biomass, product and substrate over time. Various parameters used in the mathematical modeling to be varied and it has checked the impact of changes of these parameters in the response of the fermentation process was considered. It was possible to evaluate how fast reached equilibrium and dynamic behavior of the concentrations of the variables of interest.

The tool proves to be effective in checking the parameters considered and facilitates the understanding of chosen process and assists in the interpretation of results of problems through critical analysis to solve challenges. It is a facilitator of applying basic concepts learned by the user and powerful alternative for its use of convenience and speed in achieving results.

Keywords: Computational tools; MATLAB ${ }^{\circledR}$; Bioreactor.

\section{Referências bibliográficas}

BAILEY, J. E.; OLLIS, D. F., Biochemical Engineering Fundamentals, $2^{\text {nd }}$ edition, McGraw-Hill, New York: 1986.

BELHOT, R. V.; FIGUEIREDO, R. S.; MALAVÉ, C. O., O Uso da Simulação no Ensino de Engenharia, in: Anais do XXIX Congresso Brasileiro de Ensino de Engenharia, Porto Alegre: 2001.

BORZANI, W.; AQUARONE, E.; LIMA, U. A.; SCHMIDELL, W. Biotecnologia Industrial - Processos Fermentativos e Enzimáticos, Edgard Blucher LTDA, São Paulo: 2001.

BREITKREITZ, M. C.; SOUZA, A. M.; POPPI, R. J., Experimento didático de quimiometria para planejamento de experimentos: avaliação das condições experimentais na determinação espectrofotométrica de ferro II com o-fenantrolina. Um tutorial: Parte III, Química Nova, v. 37, n. 3, p. 564-573, 2014. 
CONDORENA, E. G. B., Identificação de processos e controle preditivo com modelo utilizando técnicas de inteligência artificial aplicadas à produção de bioetanol, Tese, Faculdade de Engenharia Química, Universidade Estadual de Campinas - UNICAMP, 2012.

DIAS, R. V.; VASCONCELOS, L. G. S.; BRITO, R. P., On the Necessary and Sufficient to Efficient Use of Software in the Teaching of Chemical Engineering, Modern Applied Science, v. 7, n. 3, p. 4355, 2013.

DIEHL, F. C., Análise, Controle e Otimização Operacional de um Reator de Zymomonas mobilis com Multiplicidade de Equilíbrios, Dissertação, Departamento de Engenharia Química, Universidade Federal do Rio Grande do Sul, 2009.

FOGLER, H. S., Elements of Chemical Reactor Engineering, $3^{\text {d }}$ edition, Prentice-Hall, New Jersey: 1999.

INGHAM, J., DUNN. I. J., HEINZLE, E., PRENOSIL, J. E., SNAPE, J. B., Chemical Engineering Dynamics, Wiley-VCH. New York: 2007.

JANECZKO, M. U.; KILIAN, J.; SOARES, A. B. J.; SOARES, M. B. A., estudo da modelagem da produção de carotenoides por Sporidiobolus Salmonicolor CBS 2636 em biorreator, in: Anais do XX Congresso Brasileiro de Engenharia Química, Florianópolis: 2014.

KOZMA, R. B.; RUSSELL, J.; J., Multimedia and Understanding: Expert and Novice Responses to Different Representations of Chemical Phenomena, Journal of Research in Science Teaching, $v$. 34, p. 949-968, 1997.

LEITE, J. P.; BELTRÃO, M. J. C.; CHEBAR, I. E.; RAMIREZ, N. I. B., Avaliação preliminar do impacto de softwares de simulação no ensino das Engenharias Química e de Petróleo, Engevista, v. 16, n. 1, p. 28-40, 2014.

LUYBEN, W. L., Process Modeling, Simulation and Control for Chemical Engineers, $2^{\text {nd }}$ edition, McGraw-Hill. New York: 1996.

MARIANI, V. C.; MARTIM, E., Aplicações do Matlab no ensino de disciplinas básicas nos cursos de engenharia, in: Anais do XXXI Congresso Brasileiro de Educação em Engenharia, Rio de Janeiro: 2003

MENEZES, G. D. G., Produção de Poligalacturonase pela Linhagem Aspergillus niger Mutante 3T5B8 por Fermentação Semi-Sólida em Biorreatores de Coluna, Dissertação, Departamento de Engenharia Química, Universidade Federal Rural do Rio de Janeiro - UFRRJ, 2006.

PELISSON, G. T.; VIEIRA, F. T., Intensificação do uso de ferramentas computacionais nos cursos de graduação em Engenharia Química - A experiência da UFES, Campus Alegre, in: Anais do XI Congresso Brasileiro de Engenharia Química em Iniciação Científica, Campinas: 2015.

RIBEIRO, A. A.; GRECA, I. L., Simulações computacionais e ferramentas de modelização em educação química: uma revisão de literatura publicada, Química Nova, v. 26, n. 4, p. 542-549, 2003.

SCHMIDELL, W. ; FACCIOTTI, M. C. R. Biorreatores e processos fermentativos. In: Schmidell, W.; Lima, U. A.; Aquarone, E.; Borzani, W., Biotecnologia Industrial, 1a edição, Edgard Blücher Ltda., São Paulo: 2001.

SEEBORG, D. E.; EDGAR, T. F.; MELLICHAMP, D. A., Process Dynamics and Control, $2^{\text {nd }}$ edition, John Wiley \& Sons, Danvers: 2003. 